\title{
Preparation of a Novel Amino Functionalized Fluorescein-doped Silica Nanoparticle for $\mathrm{pH}$ Probe
}

\author{
Chunjuan Xie ${ }^{1}$, Dongguang Yin ${ }^{1 *}$, Jian $\mathrm{Li}^{2}$, Li Zhang ${ }^{2}$, Binhu Liu¹, Minghong Wu1* \\ ${ }^{1}$ College of Environmental and Chemical Engineering, Shanghai University, Shanghai $200444{ }^{2}$ College of Environmental and Chemical \\ Engineering, Nanchang Hangkong University, Nanchang 330063
}

*Corresponding authors. Email: ydg@shu.edu.cn

\begin{abstract}
A novel type of amino functionalized core-shell fluorescein isothiocyanate-doped silica nanoparticles was synthesized using a simple and effective approach of reverse microemulsion. Isothiocyanate coupled with a silane coupling agent, 3-aminopropyltrimethoxysilane, was incorporated into silica sphere and the dye molecules were bound with silica sphere through hydrolysis and polymerization of tetraethoxysilane and 3-aminopropyl-trimethoxysilane. With the covalent binding between the dye molecules and silica sphere, the leakage of the dye was avoided. With amino groups on the surface, the nanoparticles can be directly conjugated with biological molecules with no need of surface modification. The nanoparticles were $\mathrm{pH}$ sensitive, highly fluorescent and highly photostable. The $\mathrm{pH}$ response range and $\mathrm{pH}$ responsive linear range were 2.8 8.5 and 4.6 8.0, respectively. More interestedly, the nanoparticles could be phagocytosed by murine neural stem cell and could be applied to detect $\mathrm{pH}$ value for single alive cell.
\end{abstract}

Keywords: Fluorescein isothiocyanate; Water in oil microemulsion; $\mathrm{pH}$ probe; 3-aminopropyl-trimethoxysilane

Citation: C. Xie, et al. Preparation of a novel amino functionalized fluorescein-doped silica fluorescent nanoparticle for $\mathrm{pH}$ probe. Nano Biomed Eng. 2009, 1(1), 27-31. DOI: 10.5101/nbe.v1i1.p27-31

\section{Introduction}

Development of studies on chemical sensors and biosensors was very rapid in the past twenty years [1, 2]. The $\mathrm{pH}$ sensor is one of the most important and widely studied sensors because $\mathrm{pH}$ measurement was closely linked to modern industry, agriculture, medicine, bioengineering, environment, disease diagnostics and scientific studies. Several methods for $\mathrm{pH}$ sensors have been developed so far [3-6]. Reverse microemulsion is one of the most widely used methods for preparation of nanoparticles $[1,7-10]$. It can be defined as a thermodynamically stable, isotropic, and transparent solution of oil, water, and surfactant, wherein the water droplets are dispersed as nanosized liquid entities in a continuous phase of oil. The nanodroplets of water serve as a nanoreactor for the synthesis of nanoparticles. This method has advantages of no requirement of extreme condition such as temperature and pressure. In addition, size and shape of the nanoparticles can be simply controlled by varying microemulsion parameters [11-15]. In comparison with traditional organic fluorescent dyes, these nanoparticle are extremely bright and stable. Results of our previous studies show that isothiocyanate (FITC) was easily to be leaked from porous network silica shell if the FITC is encapsulated in silica shell directly using the mentioned method. In present study, a novel precursor of FITC-APTMS was first prepared using a saline reagent of 3-aminopropyltrimethoxysilane (APTMS). Then a core-shell FITCdoped silica fluorescent nanoparticle was synthesized with the reverse microemulsion method. Results show that FITC leakage was tremendously reduced with present approach. Based on the fact that bare protons can penetrate the three-dimensional porous network structure of silica shell, and the nanoparticles can be swallowed by single mouse neural stem cell and, the nanoparticles have a good response to $\mathrm{pH}$, it is expected that the nanoparticles could be applied as nano-pH sensors to monitor $\mathrm{pH}$ value of single cell in real-time.

\section{Materials and Methods}

\subsection{Materials and instruments}

FITC, Triton X-100, APTMS, TEOS, n-hexanol, cyclohexane(Sigma). Mouse neural stem cells obtained from School of life sciences, Shanghai University. (Shanghai, China). Distilled and deionized water was 
used for the preparation of all aqueous solutions. All of chemicals and reagents used in this study were of analytical grade quality. JEM-2010F transmission electron microscope (Japan). SPM-9600 atomic force microscope(Japan). Hitachi U-3010 UV-Vis spectro- photometer(Japan). Hitachi F-4500 spectrofluorimeter (Japan). FV1000 laser confocal scanning microscope (Japan).

\subsection{Preparation of the nanoparticles}

Glass vessels used for experiments were cleaned with $5 \%$ HF solution and deionized water. To prepare the precursor of FITC-APTMS, $10 \mu \mathrm{L}$ APTMS and 1.5 mg FITC were dissolved in $1 \mathrm{~mL}$ of absolute ethanol and stirred magnetically for $24 \mathrm{~h}$ in darkness. To prepare the nanoparticles, the reverse microemulsion was formed by mixing $1.77 \mathrm{~mL}$ of Triton $\mathrm{X}-100,7.5 \mathrm{~mL}$ of cyclohexane, $1.8 \mathrm{~mL}$ of hexanol, and $250 \mu \mathrm{L}$ of water with vigorous stirring. After stirring for $15 \mathrm{~min}, 150 \mu \mathrm{L}$ $1 \mathrm{mg} / \mathrm{mL}$ FITC-APTMS aqueous solution and $100 \mu \mathrm{L}$ of TEOS were added to the mixture, and then $60 \mu \mathrm{L}$ $25 \%$ of $\mathrm{NH}_{3} \cdot \mathrm{H}_{2} \mathrm{O}$ was added to initiate polymerization reaction. The reaction was allowed to continue for $24 \mathrm{~h}$ at room temperature. When the reaction was completed, the nanoparticles were isolated by adding acetone to break the microemulsion and collected by centrifuging and ultrasonically washing three times with ethanol and water to remove residual surfactant and dye molecules.

\subsection{Photobleaching experiments}

To evaluate the photostability of the nanoparticles, photobleaching experiments were performed. $1 \mathrm{mg}$ of pure FITC and $1 \mathrm{mg}$ of the nanoparticles were dis-

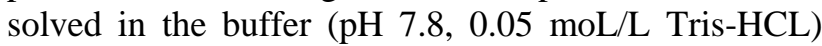
respectively, and then exposed under an excitation source of a $100 \mathrm{~W}$ xenon lamp at $518 \mathrm{~nm}$ of maximum excitation wavelength. Fluorescent intensities were recorded at every $10 \mathrm{~min}$ interval for a period of $70 \mathrm{~min}$.

\subsection{Dyeleaking experiments}

To perform dyeleaking experiments, $1 \mathrm{mg}$ of the nanoparticles was dissolved in $10 \mathrm{~mL}$ water and the mixture was dispersed by ultrasound continuously. At every $1 \mathrm{~h}, 1 \mathrm{~mL}$ of the suspension solution was taken out and centrifugated. After centrifugal separation, the precipitate was redissolved in $1 \mathrm{~mL}$ water, ultrasonic dispersed, and then fluorescent spectra were recorded. According to varies of the fluorescent intensities with time, dyeleaking of the nanoparticles in aqueous solution was evaluated.

\section{$2.5 \mathrm{pH}$ response experiments}

The nanoparticles were dispersed into water by ultrasound. $300 \mu \mathrm{L} 1 \mathrm{mg} / \mathrm{mL}$ of the nanoparticle suspension solution was mixed with $1 \mathrm{~mL} 0.05 \mathrm{~mol} / \mathrm{L}$ hosphate buffer solution with different $\mathrm{pH}$ values, incubated for $5 \mathrm{~min}$ at room temperature, and then fluo- rescent spectra and fluorescence intensities were measured.

\subsection{Cell imaging experiments}

Suspension solution of mouse neural stem cell was inoculated into the cell culture bottle and incubated in a carbon dioxide incubator, overnight $\left(5 \% \mathrm{CO}_{2}, 37^{\circ} \mathrm{C}\right)$, then washed with $0.05 \mathrm{moL} / \mathrm{L} \mathrm{PBS}(\mathrm{pH} 7.4)$ for three times. $100 \mu \mathrm{L} 1 \mathrm{mg} / \mathrm{mL}$ of the pasteurized fluorescent nanoparticle suspension solution was added into the mouse neural stem cell culture bottle containing $1 \mathrm{~mL}$ of culture medium. After incubating $15 \min \left(5 \% \mathrm{CO}_{2}\right.$, $37^{\circ} \mathrm{C}$ ) in carbon dioxide incubator and washing with the PBS buffer for three times, the cell fluorescence imaging was measured with FV1000 confocal laser scanning microscope.Fluorescence spectra and imaging of the cells were measured with different $\mathrm{pH}$ values. The $\mathrm{pH}$ values of the cells were adjusted by varying $\mathrm{pH}$ values of the culture solutions.

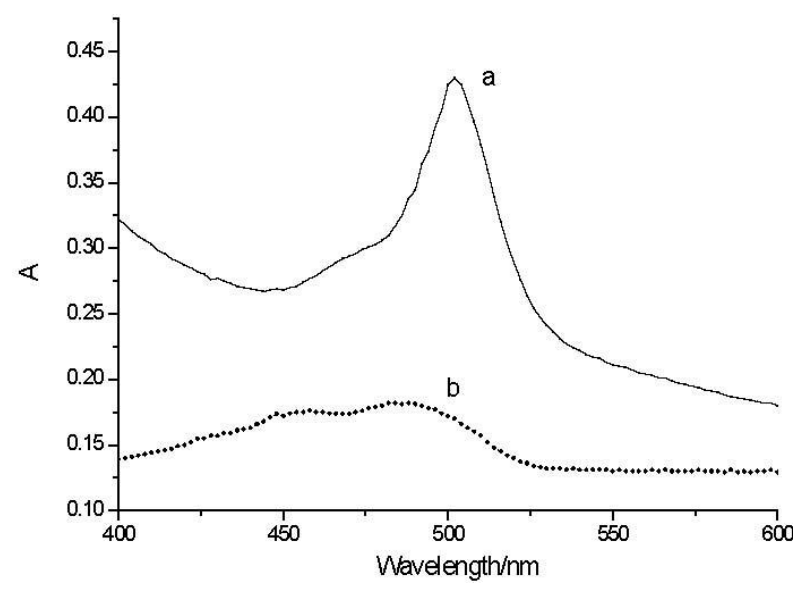

Figure 1. Ultraviolet-visible absorption spectra of (a) the nanoparticles and (b) pure FITC

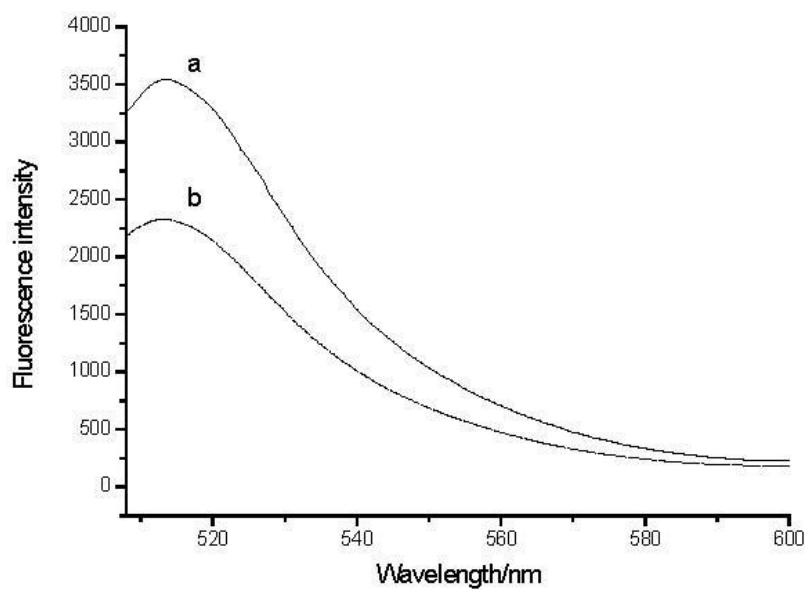

Figure 2. Fluorescence spectra of (a) the nanoparticles and (b) pure FITC 


\section{Results and discussion}

3.1 UV-visible spectroscopy and fluorescence spectra of the nanoparticles

$1 \mathrm{mg}$ of pure FITC and $1 \mathrm{mg}$ of the nanoparticles were dissolved in $2 \mathrm{~mL}$ water respectively, and then $\mathrm{UV}$-visible spectroscopy and fluorescence spectra were measured respectively. As shown in Figure 1, the absorption spectra of the pure FITC and the nanoparticles display a same profile in aqueous solution. But the absorption peak of the nanoparticles was significantly narrow and its maximum absorption wavelength was shifted $6 \mathrm{~nm}$ toward the longer wavelength compared with the pure FITC. As shown in Figure 2, their emission spectra also show a same profile but maximum emission wavelength of the nanoparticles has $5 \mathrm{~nm}$ of red-shift.

\subsection{TEM and AFM images of the nanoparticles}

Size and shape of the nanoparticles were measured

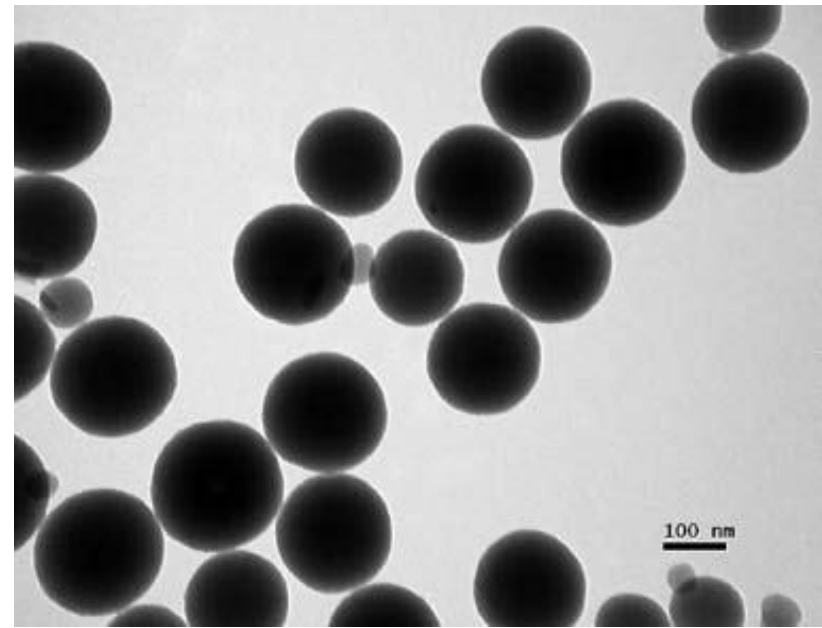

Figure 3. Transmission electron microscope (TEM) of the nanoparticles

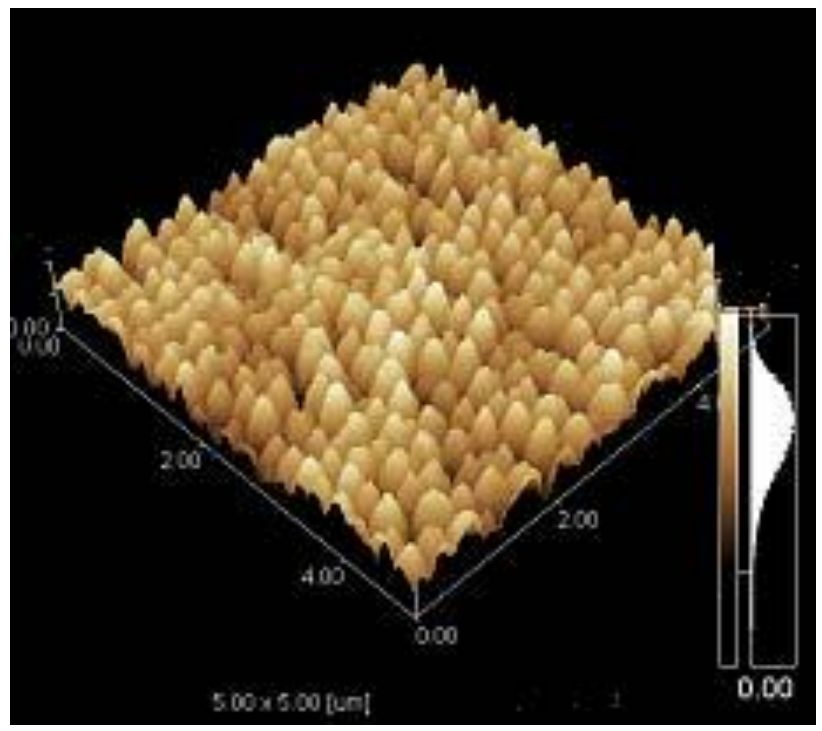

Figure 4. Atomic Force Microscope image of the nanoparticles.

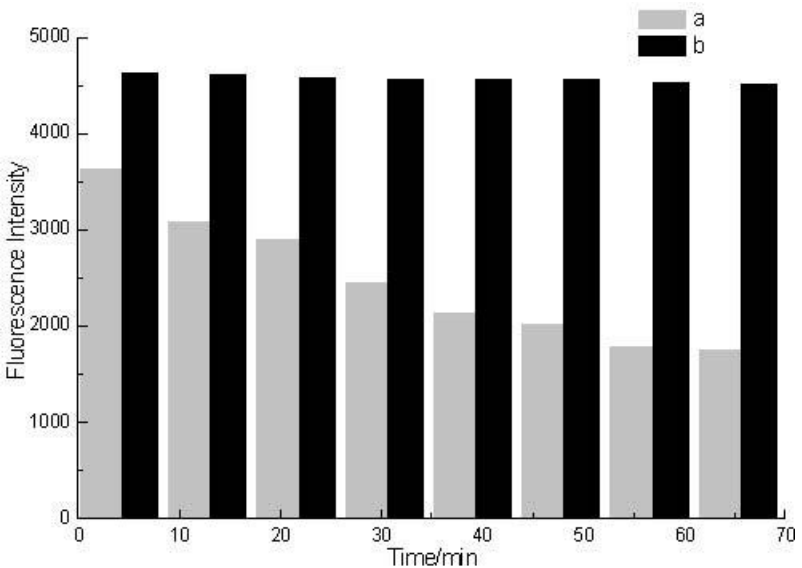

Figure 5. Photostability curve of pure FITC (a) and the nanoparticles (b)

with TEM and AFM. As shown in Figure 3 and Figure 4 , the nanoparticles are spherical, monodisperse, and uniform in shape and size with average diameter of $148 \pm 15 \mathrm{~nm}$.

\subsection{Photostability of the nanoparticles}

As shown in Figure 5, the fluorescent intensity of pure FITC was decreased approximately $51 \%$ after 70 min of continuous excitation, whereas the fluorescent intensity of the nanoparticles was only decreased $5 \%$. The high photostability of the nanoparticles is due to the fact that the FITC dye in the nanoparticles is coated surroundingly by silica, which isolates the dye molecules from the outside environment such as solvent molecules, oxygen and free radicals caused by light exposure, and effectively protects the dye molecules from photodecomposition.

\subsection{Dyeleaking of the nanoparticles}

For comparison, we used the same method to synthesize pure FITC-doped silica nanoparticles in which FITC was directly encapsulated in silica shell without precursor of FITC-APTMS. As shown in Figure 6, the fluorescent intensity of the nanoparticles was only decreased approximately $7 \%$ after continuously ultrasonic $6 \mathrm{~h}$ in aqueous solution, whereas the fluorescent intensity of the pure FITC-doped nanoparticles was decreased $42 \%$. In this approach, FITC was covalent bound to silica shell when the APTMS was copolymerized with tetraethoxysilane(TEOS) initiating by $\mathrm{NH}_{3} \bullet \mathrm{H}_{2} \mathrm{O}$. Since the FITC was covalent bound with silica shell, it tremendously reduced FITC leaking from the silica matrix. Therefore, the chemical stability of the nanoparticles in aqueous was improved significantly.

\subsection{Confirmation of amino groups on surface of the nanoparticles}

When ninhydrin reacts with amino compound, a blueviolet compound is produced with an absorb peak at $568 \mathrm{~nm}$ [16]. Based on this fact, confirmation of $\mathrm{NH}_{2}$ 
nanobe.org C. Xie et al.

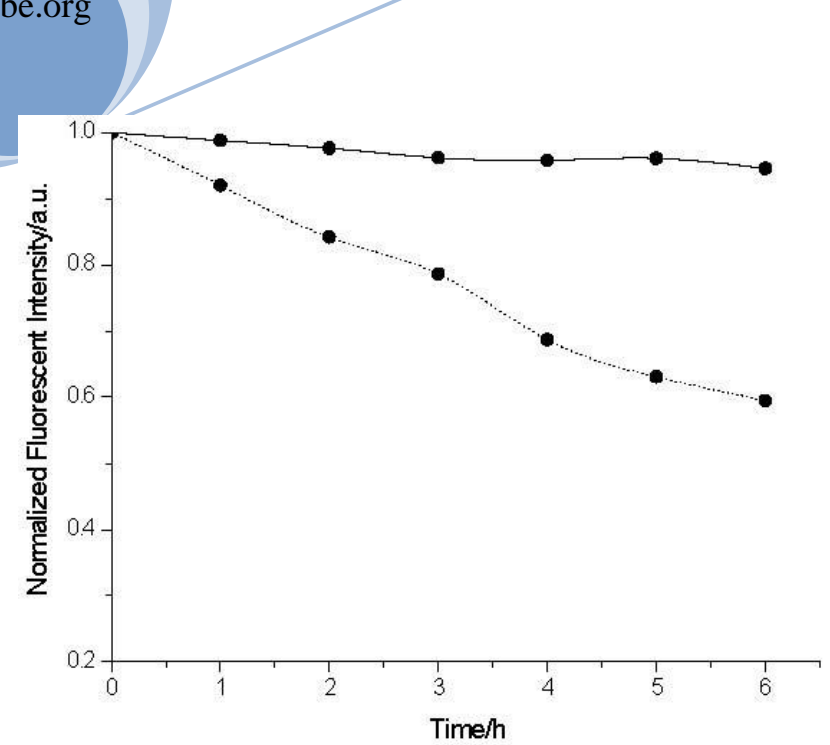

Figure 6. Fluorescent leakage curve of the nanoparticles (bold) and pure FITC-doped silica nanoparticles (dotted)

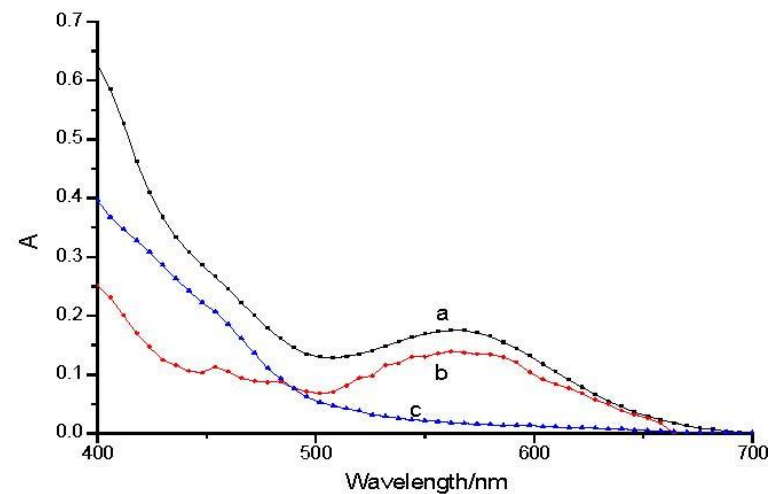

Figure 7. Utraviolet-visible (UV-VIS) absorption spectra of nanoparticles-APTMS (a), APTMS (b) and nanoparticles(c)

groups on surface of the nanoparticles was investigated. Pure APTMS and two types of the nanoparticles were compared. One type of the nanoparticles was prepared with APTMS and TEOS copolymerization and another was prepared through polymerization reaction of TEOS but without APTMS. As shown in Figure 7, pure APTMS and the former nanoparticles all have maximal absorb peaks at $568 \mathrm{~nm}$, whereas the nanoparticles prepared without APTMS have no absorption. Results demonstrated that amino groups had been introduced to the surface of the nanoparticles directly during the preparation process. Since the nanoparticles have active amino groups on their surface, they can be directly used to conjugate biological molecules with no need of complicated surface modification.

\section{$3.6 \mathrm{pH}$ response of the nanoparticles}

Results of $\mathrm{pH}$ response curves were as shown in Figure 8 . The results show that the fluorescent intensities were increased with increasing $\mathrm{pH}$ values of PBS buffer in the experimental range of $2.8 \sim 8.5$, where $\mathrm{pH}$ responsive linear range are 4.6 8.0.

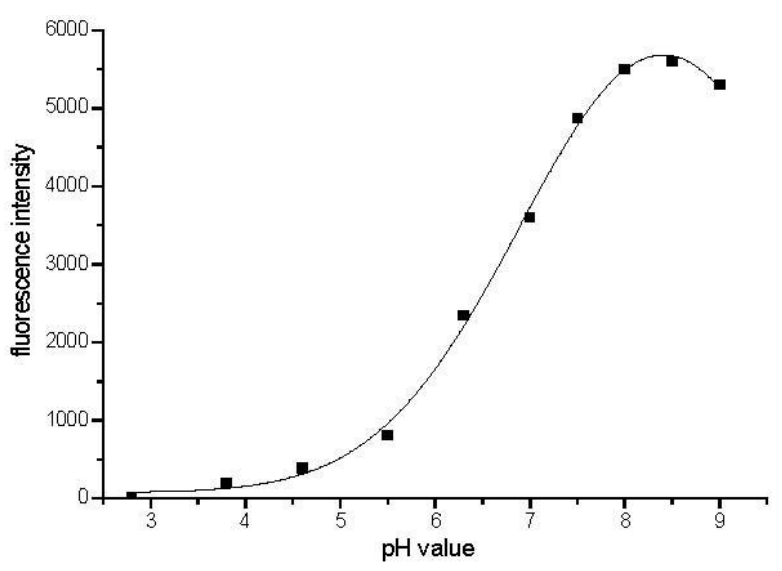

Figure 8. Fluorescent spectra of the nanoparticles against $\mathrm{pH}$ values

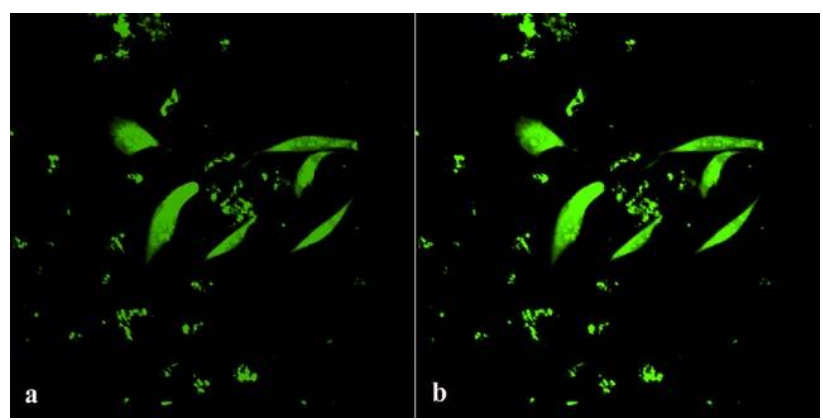

Figure 9. Fluorescence images of neural stem cells phagocytized nanoparticles at diffrernt $\mathrm{pH}$ values (a pH 6.2 b pH 7.5)

\subsection{Cell imaging of the nanoparticles}

Imagings of the cells were measured with different $\mathrm{pH}$ values. Results show that the nanoparticles can be phagocytosed by murine neural stem cells and the fluorescent intensities vary with $\mathrm{pH}$ values of the cells. As shown in Figure 9, when the $\mathrm{pH}$ values of cells were increased from 6.2 to 7.5 , the brightness and fluorescent intensity of the cells were increased correspondingly. These results indicate that the nanoparticles could be applied as nano-pH sensors to monitor $\mathrm{pH}$ value of single cell in real-time.

\section{Acknowledgements}

Financial supports from the Shanghai Pujiang Program, Shanghai Nano Program (No. 0752nm024), Shanghai Leading Academic Discipline Project (No. S30109), National Natural Science Foundation of China (No. 40830744) and Graduate Student Innovation Fund of Shanghai University (No. SHUCX091034) are gratefully acknowledged.

\section{References}

1. Chang S, Liu L, Asher S. Preparation of properties of tailored morphology, monodisperse colloidal silica-Cadmium sulfide nanocomposites. J Am Chem Soc 1994; 116(15): 6739-6744. doi:10.1021/ja00094a032 
2. Duan J, Wang K, Tan W, He X, He C. A study of a novel organic fluorescent core-shell nanoparticle. Chem J Chin Univ 2003; 24: 255-259.

3. .Han J, Cui D, Li Y, Zhang H, Huang Y, Zheng Z.Preparation of a gastroesophageal tract $\mathrm{pH}$ sensor based on the H+-ISFET and the monitoring system for $24 \mathrm{~h}$. Sens Actuators B Chem 2000; 66: 203-204 doi:10.1016/ S0925-4005(00)00363-4

4. Wang D, Chen W, Wang M, Yu D, Wang G. An on-line pH monitoring system for flow solution analysis with $\mathrm{pH}-$ ISFETs and flow-through cell. Sens Actuators B Chem 2000; 66: 6-8. doi:10.1016/S0925-4005(99)00445-1

5. Ruan C, Ong K, Mungle C, Paulose M, Nickl N, Grimes C. A wireless $\mathrm{pH}$ sensor based on the use of salt-independent micro-scale polymer spheres. Sens Actuators B Chem 2003; 96: 61-69. doi:10.1016/S0925-4005(03)00486-6

6. Shiojiri S, Hirai T, Komasawa I. Immobilization of semiconductor nanoparticles formed in reverse micelles into polyurea via in situ polymerization of diisocyanates. Chem Commun 1998; 14: 1439-1440. doi:10.1039/ a802588f

7. Stathatos E, Lianos P, Delmonte F, Levy D, Tsiourvas D.Formation of $\mathrm{TiO} 2$ nanoparticles in reverse micelles and

8. their deposition as thin films on glass substrates. Langmuir 1997; 13(16): 4295-4300. doi:10.1021/la9701 $\underline{642}$

9. Kreuter J. Nanoparticle-based drug delivery system. Control Release 1991; 16(1): 169-176. doi:10.1016/ 01683659(91)90040-K

10. Santra S, Zhang P, Wang K, Tapec R, Tan W. Conjugation of biomolecules with luminophore-doped silica nanoparticles for photostable biomarkers. Anal Chem 2001; 73(20): 4988-4993. doi:10.1021/ac010406+

11. Bentivegna F, Canva M, Georges P, Brun A, Chaput F, Malier L, Boilot J. Reverse saturable absorption in solid xerogel matrices. Appl Phys Lett 1993; 62(15):17211723. doi:10.1063/1.109585

12. Zhao X, Tapec-Dytioco R, Wang K, Tan W. Collection of trace amounts of DNA/mRNA molecules using genomagnetic nanocapturers. Anal Chem 2003; 75(14): 34763483. doi: $10.1021 / \mathrm{ac} 034330 \mathrm{o}$

13. .Bagwe R, Khilar K. Effects of the intermicellar exchange rate and cationson the size of silver chloride nanoparticles formed in reverse micelles of AOT. Langmuir 1997; 13(24): 6432-6438. doi:10.1021/la9700681

14. Bagwe R, Khilar K. Effects of the intermicellar exchange rate on the formation of silver nanoparticles in reverse microemulsions of AOT. Langmuir 2000; 16(3): 905-910. doi:10.1021/la980248q

15. Zhang P, Gao L. Copper sulfide flakes and nanodisks. Mater Chem 2003; 13(8): 2007-2010. doi:10.1039/b305 $\underline{584 \mathrm{a}}$

16. Taton T, Mirkin C, Letsinger R. Scanometric DNA array detection with nanoparticle probes. Science 2000; 289(8): 1757-1760. doi: 10.1126/science.289.5485.1757

17. Henry A, Tutt T, Galloway M. Surface modification of poly(methyl methacrylate) used in the fabrication of microanalytical devices. Anal Chem 2000; 72(21):5331-5337. doi:10.1021/ac0006851

Received 10 November, 2009; accepted 6 December, 2009; published online 9 December, 2009.

Copyright: (c) 2009 C. Xie et al. This is an open-access article distributed under the terms of the Creative Commons Attribution License, which permits unrestricted use, distribution, and reproduction in any medium, provided the original author and source are credited. 